\title{
A multimarker qPCR platform for the characterisation of endometrial cancer
}

\author{
ANNA SUPERNAT ${ }^{1}$, SYLWIA ŁAPIŃSKA-SZUMCZYK ${ }^{2}$, HANNA MAJEWSKA ${ }^{3}$, JACEK GULCZYŃSKI ${ }^{4}$, \\ WOJCIECH BIERNAT ${ }^{3}$, DARIUSZ WYDRA ${ }^{2}$ and ANNA J. ŻACZEK ${ }^{1}$ \\ ${ }^{1}$ Department of Medical Biotechnology, Intercollegiate Faculty of Biotechnology, University of Gdańsk and \\ Medical University of Gdańsk, Dębinki 1, 80-211 Gdańsk; ${ }^{2}$ Department of Gynaecology, Gynaecological Oncology \\ and Gynaecological Endocrinology, Medical University of Gdańsk, Kliniczna 1a, 80-402 Gdańsk; \\ ${ }^{3}$ Department of Pathomorphology, Medical University of Gdańsk, Dębinki 7, 80-211 Gdańsk; ${ }^{4}$ Department of \\ Pathology and Neuropathology, Medical University of Gdańsk, Dębinki 1, 80-211 Gdańsk, Poland
}

Received September 25, 2013; Accepted November 20, 2013

DOI: 10.3892/or.2013.2924

\begin{abstract}
The molecular background of endometrial cancer (EC) has not been fully elucidated. In the present study, we developed a quantitative PCR (qPCR) platform to examine the gene dosages of the potential molecular markers $M G B 1$, TOP2A, ERBB1-4, MYC, CCND1, ESRI and PI3K. The platform was applied in samples collected from $157 \mathrm{EC}$ patients (stage I-IV) to verify its clinical utility and to examine the diagnostic and prognostic significance of the analysed biomarkers. The gene dosage pattern of the $E R B B$ family and its downstream effectors $P I 3 K$ and $M Y C$ showed particularly strong correlations with clinicopathological data. The ERBB PI3K/Akt pathway was upregulated in $31(20 \%)$ of 156 cases. Activation of the ERBB PI3K/Akt pathway was positively correlated with a higher stage $(\mathrm{p}=0.001)$, higher grade $(p=0.001)$, histological type II disease $(p=0.0003)$ and metastases $(\mathrm{p}=0.02)$. The implemented hierarchical clustering revealed that cluster 2 was characterised by high copy numbers of the studied genes. Cluster 2 was associated with shorter overall survival $(\mathrm{p}=0.05)$. The platform was found to be a fast and simple method for direct analysis of the genes involved in uterine carcinogenesis, making it feasible for EC biology characterisation.
\end{abstract}

\section{Introduction}

Endometrial cancer (EC) is the most frequent malignancy of the female genital tract in the Western world, with an estimated

Correspondence to: Dr Anna J. Żaczek, Laboratory of Cell Biology, Department of Medical Biotechnology, Intercollegiate Faculty of Biotechnology, University of Gdańsk and Medical University of Gdańsk, Dębinki 1, 80-211 Gdańsk, Poland

E-mail: azaczek@gumed.edu.pl

Key words: ERBB PI3K/Akt pathway, molecular markers, quantitative PCR platform, endometrial cancer incidence of 10-20 per 100,000 women (1). There were 5,594 new cases and 1,028 deaths estimated in 2012 in Poland (2). The prevalence of EC is increasing annually (3). Despite the high prevalence, our understanding of the molecular background of EC with regard to its pathogenesis, tumour growth and disease progression remains insufficient. Data concerning copy number variations within EC are scarce.

EC encompasses a group of histologically and biologically diverse tumours, which are characterised by a distinct pathogenesis. Two pathogenic types have been distinguished based on the differential characteristics, as proposed by Bokhman (4). This classification has been challenged in daily practice, as some tumours possess overlapping or combined morphologic and molecular characteristics. Nevertheless, the dualistic model is still followed in clinical practice (5), with endometrial carcinomas being classified as either type I or II.

The more frequent endometrioid type I carcinomas (80\%) (6) are rather well-differentiated, with high oestrogen receptor $\alpha(\mathrm{ER} \alpha)$ and progesterone receptor (PR) expression. They are generally confined to the uterus and carry a favourable prognosis. Type II carcinomas, which have non-endometrioid serous or clear cell histology, follow an oestrogen-unrelated pathway. Such poorly-differentiated tumours behave in a more aggressive manner, resulting in a poor prognosis $(4,7,8)$. Type II carcinomas appear spontaneously and they are not clearly related to the transition from atypical hyperplasia; rather, they arise in the background of an atrophic or inert endometrium (9).

Type I endometrioid carcinomas have a near-diploid karyotype and are associated with microsatellite instability, which is the consequence of alterations in the mismatch repair genes, MLH1, MSH6 and MSH3. Aberrations of K-RAS oncogene together with tumour-suppressor gene mutations, especially PTEN, have also been reported. Additionally, type I ECs contain PI3K and $\beta$-catenin mutations. On the other hand, type II serous carcinomas are known for their chromosome aneuploidy, $E R B B 2$ overexpression and mutant p53 protein accumulation within the nucleus. Other mutations, encountered in both types of EC, include aberrations of $M Y C, I N T 2$ and CTNNBI (6,10-15). 
The prognostic value of the EC dualistic model is limited. Up to 20 and $50 \%$ of type I and II carcinomas, respectively, recur (16). The aim of the present study was to further explore the molecular basis of EC by developing a quantitative PCR (qPCR) platform that would allow for more precise tumour characterisation. We searched for potential molecular markers that may be of diagnostic and prognostic significance.

We analysed the RNA expression level of the $M G B 1$ gene (mammaglobin), as this secretory protein is recognised as a sensitive marker in breast cancer and was also found to be expressed in the female genital tract and in EC (17). We also measured the copy number variations of the following genes: $E S R 1$ (oestrogen receptor 1), ERBBI (epidermal growth factor receptor), $E R B B 2$ (v-erb-b2 erythroblastic leukaemia viral oncogene homolog 2, also known as HER2), ERBB3 (receptor tyrosine-protein kinase erbB-3), ERBB4 (receptor tyrosineprotein kinase erbB-4), PI3K (phosphatidylinositol 3-kinases), $M Y C$ (cellular homolog of the retroviral v-myc oncogene), CCND1 (cyclin D1) and TOP2A (DNA topoisomerase 2- $\alpha$ ).

ERBB2 is a central oncogene of prognostic and predictive value. Its amplification and increased expression have been reported in EC, indicating a higher stage and poor differentiation (18). Previous reports indicated the importance of the interaction of ERBB2 with other ERBB receptors (ERBB1 ERBB3, ERBB4). This interaction may be associated with a more aggressive phenotype $(19,20)$. PI3K and MYC are downstream effectors of ERBB receptors. Excessive activation of PI3K, as a result of amplification, is associated with a poorer prognosis in EC patients (21). Expression of the $M Y C$ oncogene is often impaired in several types of cancer and gene amplification is one of the mechanisms of its activation (22). Another gene implicated in endometrial carcinogenesis is CCNDI; its amplification may result in cyclin D1 overexpression, which is found in $40-56 \%$ of EC cases (23). TOP2A is an enzyme that plays a key role in maintaining the integrity of the genome. In addition, TOP2A is a target of many anticancer drugs. Amplification and overexpression of TOP2A has been observed in 7.5 and $45 \%$ of EC cases, respectively (24).

The gene dosage of the aforementioned genes seems to be of clinical importance in EC. However, these genes have not been examined together in EC. Therefore, we developed a potentially highly sensitive and reproducible qPCR-based platform for the reliable assessment of specific genes associated with EC and in order to analyse its utility in a representative group of EC samples.

\section{Materials and methods}

Patients and tissues. The present study included 157 fresh frozen tumour samples retrospectively collected from a cohort of EC patients who were operated on in the Department of Gynaecology, Gynaecological Oncology and Gynaecological Endocrinology (Medical University of Gdańsk) between 2005 and 2011. Each patient was primarily treated by surgery, with the possible option of radiotherapy and/or chemotherapy administration. The inclusion criteria were operable EC (IVB stage patients underwent cytoreductive surgery) confirmed by histological examination and a signed consent form. The present study was approved by the Ethics Committee of the Medical University of Gdańsk.
The samples were collected from the core of the tumour by surgical excision prior to any systemic treatment and were immediately frozen and stored at $-80^{\circ} \mathrm{C}$. The samples were kept on ice during transport. The tissue samples included all pathologic stages of endometrial carcinoma, from non-invasive IA to metastatic IVB cancer as distinguished by the International Federation of Gynecology and Obstetrics (FIGO) in 2009 (25). The patient characteristics are summarized in Table I. The mean age was 63.6 years (range 30-87 years). Patients with a body mass index $>30$ were classified as obese (26). The PgR, ER and ERBB2 status was determined immunohistochemically (IHC) using Allred score (positivity cut-off, $\geq 3$ ) for PgR and ER and the HercepTest score (positivity cut-off, 3) for ERBB2. A survival analysis was performed for 82 patients. After a median follow-up of 4.4 years (range 0.04-7.58 years), 15 patients $(18 \%)$ had died. The last follow-up data were collected in January 2013.

Controls. Healthy endometrium from patients treated for ailments other than EC was collected as control samples. Control samples were accessed histopathologically; no hyperplasia was observed. Pooled DNA and RNA was isolated from frozen tissue samples of five healthy donors and used for qPCR assay optimisation, standard curve generation and as a calibrator for the gene dosage and expression evaluation test in 157 EC tumour samples.

The utility of the assay was verified using the human cancer cell line SK-BR-3 with confirmed amplification of the genes ERBB2, MYC, TOP2A and human cancer cell line MDA-MB-361 with confirmed amplification of ERBB2 (27). Additionally, we used reference DNA (Roche, Switzerland) and DNA isolated from healthy breast tissue to verify the performance of the EC DNA.

DNA and RNA isolation. Prior to nucleic acid isolation, tissue specimens $(25 \mathrm{mg} / \mathrm{sample})$ were homogenised (1 min, 6,000 rpm speed) using a MagNA Lyser (Roche). DNA and RNA were isolated with AllPrep DNA/RNA Mini kit (Qiagen, Germany) according to the manufacturer's instructions. After the isolation, the DNA/RNA concentration and purity were determined using a Spectrophotometer ND-1000 (NanoDrop, USA). Good quality of the DNA was defined as an A260/280 nm ratio between 1.70 and 1.90. Good quality of the RNA was defined as an A260/280 nm ratio of $~ 2$. The DNA and RNA samples were stored at -20 and $-80^{\circ} \mathrm{C}$, respectively.

RNA was subsequently reverse transcribed to cDNA with the Transcriptor First Strand cDNA synthesis kit (Roche) according to the manufacturer's instructions using random hexamer primers. The total amount of 1,000 ng RNA was used per each reverse transcription reaction.

Genes and primers. The gene dosages of ERBB1, ERBB2, ERBB3, ERBB 4, MYC, CCND1, ESR I, PI $3 K$ and TOP2A were determined by qPCR with Power SYBR-Green Mastermix (Applied Biosystems, USA), using the amyloid precursor protein $(A P P)$ gene and $3 P$ (RNA, U4 small nuclear pseudogene) gene as a reference. The potential reference genes were chosen based on a search performed in the Atlas of Genetics and Cytogenetics in Oncology and Haematology (http://www. atlasgeneticsoncology.org/). $A P P$ and $3 P$ stability was 
Table I. Clinicopathological data $(\mathrm{N}=157)$.

\begin{tabular}{ll}
\hline Variable $\quad$ No. of cases $(\%)$
\end{tabular}

\begin{tabular}{|c|c|}
\hline \multicolumn{2}{|l|}{ Menopausal status } \\
\hline Premenopausal & $8(5.1)$ \\
\hline Perimenopausal & $11(7)$ \\
\hline Postmenopausal & $124(79)$ \\
\hline Missing data & $14(8.9)$ \\
\hline \multicolumn{2}{|l|}{ Histology } \\
\hline Endometroid & $123(78.3)$ \\
\hline Non-endometroid & $34(21.7)$ \\
\hline \multicolumn{2}{|l|}{ Stage $\left(\mathrm{FIGO}^{\mathrm{a}}\right)$} \\
\hline IA-IB & $112(71.3)$ \\
\hline II & $22(14)$ \\
\hline IIIA-IIIC & $20(12.7)$ \\
\hline IVA-IVB & $3(1.9)$ \\
\hline \multicolumn{2}{|l|}{ Grade } \\
\hline I & $60(38.2)$ \\
\hline II & $69(43.9)$ \\
\hline III & $24(15.3)$ \\
\hline Missing data & $4(2.5)$ \\
\hline \multicolumn{2}{|l|}{ Obesity } \\
\hline Absent & $68(43.3)$ \\
\hline Present & $87(55.4)$ \\
\hline Missing data & $2(1.3)$ \\
\hline \multicolumn{2}{|l|}{ Cervical invasion } \\
\hline Absent & $113(72)$ \\
\hline Present & $42(26.8)$ \\
\hline Missing data & $2(1.3)$ \\
\hline
\end{tabular}

Myometrial infiltration

$\begin{array}{lc}\leq 1 / 2 & 87(55.4) \\ >1 / 2 & 69(43.9) \\ \text { Missing data } & 1(0.6)\end{array}$

Metastases

Absent

105 (66.9)

Present

Missing data

3 (1.9)

PgR status

Negative

12 (7.6)

Positive

Missing data

$51(32.3)$

ER status

Negative

Positive

Missing data

ERBB2 status

Negative

$89(56.7)$

Positive

$17(10.8)$

Missing data verified against superoxide dismutase 2 (SOD2) using geNorm software (28). The primers were designed using Beacon Designer Software version 7.6 (Bio-Rad, USA). Their specificity was examined by PCR, followed by electrophoretic separation on $1.5 \%$ agarose gel stained with ethidium bromide (final concentration of $0.5 \mu \mathrm{g} / \mathrm{ml}$ ). Electrophoresis was performed in $0.5 \mathrm{X}$ TBE buffer at $100 \mathrm{~V}(5 \mathrm{~V} / \mathrm{cm})$ for $30 \mathrm{~min}$. For visualisation of the bands, the gel was placed in a Molecular Imager ${ }^{\circledR}$ GelDoc $^{\mathrm{TM}}$ XR photoimaging system and analysed using Quantity-One software (both from Bio-Rad) after irradiation with UV light (302 nm). The primer parameters are summarised in Table II. The quantity of DNA/well was $40 \mathrm{ng}$.

The MGB1 RNA expression level was determined by RT-qPCR with TaqMan ${ }^{\circledR}$ Universal PCR Master Mix (Applied Biosystems), using hypoxanthine phosphoribosyltransferase 1 (HPRT1) as a reference. The stability of the HPRT1 gene expression was verified relative to the expression of glyceraldehyde 3-phosphate dehydrogenase (GAPDH) and $\beta$-actin $(A C T B)$. We used TaqMan ${ }^{\circledR}$ Expression Assays (Applied Biosystems) as follows: HPRT1 Endogenous Control Hs99999909_m1; MGB1 Gene Expression Assay Hs00935948_m1. The quantity of cDNA/well was $75 \mathrm{ng}$.

Calculating the optimal primer concentration. The optimal forward/reverse primer concentration was based on determination of the minimum quantification cycle $(\mathrm{Cq})$ and the maximum $\Delta R_{n}$ while minimizing nonspecific amplification. The reaction volumes were $20 \mu \mathrm{l}$. We used $40 \mathrm{ng}$ of genomic DNA as a template. Each of the nine forward/reverse primer concentration combinations $(200 / 200,300 / 200$, 400/200, 200/300, 300/300, 400/300, 200/400, 300/400 and $400 \mathrm{nM} / 400 \mathrm{nM}$ ) was run in triplicate. Quantities of gene expression assays and TaqMan ${ }^{\circledR}$ Universal PCR Master Mix were used according to the manufacturer's instructions.

Assessment of gene dosage and mRNA expression. After the optimisation, the components of the prepared mastermix in the final protocol used for qPCR standard curve generation as well as ERBB1, ERBB2, ERBB3, ERBB4, MYC, CCND1, ESR 1, PI3K and TOP2A gene dosage determination in $157 \mathrm{EC}$ tumour samples were: $10 \mu \mathrm{l}$ of $2 \mathrm{X}$ Power SYBR-Green Mastermix, 0.4-0.6 $\mu \mathrm{l}$ of $10 \mu \mathrm{M}$ forward/reverse primer (Sigma-Aldrich, Germany), $40 \mathrm{ng}$ of DNA in the volume of $4 \mu \mathrm{l}$ and water added into the reaction mixture to obtain a final volume of $20 \mu 1$.

Reactions amplifying $E R B B 1, E R B B 2, E R B B 3, E R B B 4$, $M Y C, C C N D 1, E S R 1, P I 3 K, T O P 2 A$ and two reference genes $(A P P$ and $3 P$ ) were performed in separate wells. The reactions were always prepared in duplicate on 96 -well plates and sealed with optical tape (both from Applied Biosystems). For each tested gene, a negative control (no template DNA) was always included. A melting curve analysis was also included in all gene dosage determinations.

Reactions amplifying MGB1 and HPRT1 cDNA were performed in separate wells. The reactions were always prepared in triplicate on 96-well plates and sealed with optical tape (both from Applied Biosystems). For each tested gene, a negative control (RNA and reverse transcription mix without reverse transcriptase) was always included.

${ }^{\mathrm{a}}$ FIGO, International Federation of Gynecology and Obstetrics. 
Table II. Primer parameters.

\begin{tabular}{llcccc}
\hline Name & \multicolumn{1}{c}{ Sequence 5'>3' } & $\begin{array}{c}\text { No. of } \\
\text { base pairs }\end{array}$ & $\begin{array}{c}\text { Product } \\
\text { length (bp) }\end{array}$ & $\begin{array}{c}\text { Reference } \\
\text { sequence }\end{array}$ & $\begin{array}{c}\text { Final primer } \\
\text { concentration (nM) }\end{array}$ \\
\hline $3 P$ & F: CTC ATA GGC GAA GGC ACC AG & 20 & 112 & NT_022517 & 300 \\
$3 P$ & R: GGT CAA GTT CCG CAC ACA CC & 20 & & & 300 \\
APP & F: AGC CCA GAA GGT GTC AAA CA & 20 & 60 & NG_007376 & 300 \\
APP & R: CAT CTT CAT GTC CGT TGC AT & 20 & & & 300 \\
CCND1 & F: AGG AGG TGT GAG GAG GAG & 18 & 102 & NG_007375 & 300 \\
CCND1 & R: CTG GAA GTC AAC GGT AGC & 18 & & & 300 \\
$M Y C$ & F: CGT GAC CAG ATC CCG GAG TT & 20 & 134 & NG_007161 & 300 \\
$M Y C$ & R: CGT TTC CGC AAC AAG TCC TCT & 21 & & & 200 \\
ERBB1 & F: GCA GAC AGG ATG ACC AAG AG & 20 & 91 & NG_007726 & 300 \\
ERBB1 & R: GTA GGC AGA TGA ACA GGA ACC & 21 & & & 300 \\
ERBB2 & F: CTG CTG GTC GTG GTC TTG G & 19 & 93 & NG_007503 & 300 \\
ERBB2 & R: CTG CAG CAG TCT CCG CAT C & 19 & & & 300 \\
ERBB3 & F: GAG AGG TGT GAG GTG GTG ATG G & 22 & 114 & NG_011529 & 300 \\
ERBB3 & R:AAG AGG AGC AGG TTG AGG AAG G & 22 & & & 300 \\
ERBB4 & F: CGC TTC GTC TCT TCT CGT TTC C & 22 & 90 & NG_011805 & 300 \\
ERBB4 & R: GAA CAA CAA TGG CAC GCT AAT CC & 23 & & & 200 \\
ESR1 & F: ACA TGG ACA CCT CCC AGT C & 19 & 60 & NG_008493.1 & 300 \\
ESR1 & R: ACA GAC TAA CAC AGC CCA TC & 20 & & & 300 \\
PI3K & F: GCT TCC AAC AAT CCT CTT CCG TAG & 24 & 118 & NG_012113.1 & 200 \\
PI3K & R: GCA CTG AAT CTG TAG CGA ACT TCC & 24 & & & 300 \\
$T O P 2 A$ & F: GCC AAC TCA GCC GTT CAT AGG & 21 & 101 & NG_027678 & 200 \\
TOP2A & R: CGA AGC AGA CCA GCC AAT CC & 20 & & & 200 \\
\hline
\end{tabular}

The thermal profiles used were the default settings of the manufacturer for either SYBR-Green or TaqMan ${ }^{\circledR}$ probe assays. Reports containing the $\mathrm{Cq}$ values were generated using StepOne Software v2.2.2 (Applied Biosystems).

Assay evaluation and quality control. To overcome the shortcomings of the in-built StepOnePlus ${ }^{\mathrm{TM}}$ software normally used to analyse data, we utilized qbase ${ }^{\text {PLus }}$ software, version 2.3 (Biogazelle, Belgium) (29) for gene dosage and expression quantification. The software uses a modified $\Delta \Delta \mathrm{Ct}$ method based on multiple reference genes and gene specific amplification efficiencies. It also possesses an algorithm that allows for inter-run calibration, thus taking into account run-to-run variation.

Average $\mathrm{Cq}$ values from the duplicates/triplicates were used for gene dosage/mRNA expression calculation. Inter-run calibration was implemented. The reference target stability was verified, the values of $\mathrm{M}$ and $\mathrm{CV}$ were $<0.5$ and 0.2 , respectively. The targets were scaled to the average. Userdefined amplification efficiencies separate for each gene were assumed. The reaction efficiencies of $E R B B 1, E R B B 2, E R B B 3$, ERBB 4, MYC, CCNDI, ESR1, PI3K, TOP 2A, APP, 3P, MGB1 and $H P R T 1$ under the optimised conditions were calculated from the five-point standard curves prepared by amplifying the pooled control genomic DNA/cDNA, diluted over four orders of magnitude. Standard curves were automatically generated by the StepOne Software v2.2.2. Baseline cycles and thresholds were set manually and the Cqs were calculated automatically by the software using an in-built algorithm.

The integrity of the nucleic acids in randomly selected samples was checked by visualisation of the electrophoretically separated post-PCR products $(6,4 \mathrm{~kb}$ fragment of CYP2D6 gene) and when compared with the molecular marker GeneRuler ${ }^{\mathrm{TM}} 1 \mathrm{~kb}$ DNA Ladder (Thermo Scientific, USA).

The presence of the PCR inhibitors was evaluated by performing qPCR with pooled control DNA and genomic DNA from healthy breast tissue and reference DNA from Roche, followed by comparison of the Cq values. The reactions were conducted in triplicate on a test plate, with a negative control (no template DNA) included.

The reaction specificity was verified through a melt curve analysis (included in every run) and by visualisation of the electrophoretically separated random post-PCR products for all studied genes.

Statistical analysis. STATISTICA software version 10 (StatSoft Co., USA) was used for all calculations, with the exception of unsupervised hierarchical clustering. Data visualisation, dendrograms and complete linkage computations were performed with the use of the Java Development kit $7 \mathrm{u} 21$ script running in Java Runtime Environment 7. The tests that were used and their applications were: testing normality of the 
data set, Shapiro-Wilk test, correlations between continuous relative gene quantities, Spearman's correlation, correlations between continuous or categorical relative gene quantities and clinicopathological data of the patients, Mann-Whitney U test or crosstabs statistics with Pearson's Chi-square test, respectively. The Kaplan-Meier estimator was employed for survival analysis and the results were verified with the Cox F-test. The endpoint for the present study was overall survival (OS). OS was defined as the time from sample collection to mortality or censoring. Censoring was defined as loss of follow-up or alive at the end of follow-up. Statistical significance in all the aforementioned tests was assumed when $\mathrm{p} \leq 0.05$. Cox proportional hazards regression analysis was used to identify the independent predictors of OS. Univariate predictors significant with a value of $\mathrm{p} \leq 0.10$ were entered into a step-wise multivariate model to identify those with independent prognostic information. The study was performed in accordance with the REMARK criteria (30).

\section{Results}

Flow of samples. Of the 157 RNA samples, $156(99.4 \%)$ had amplifiable cDNA. Amplifiability of the DNA samples differed depending on the target, ranging from $152(97.5 \%)$ to $156(99.4 \%)$.

Assay evaluation and quality control results. The parameters of the standard curves obtained for ERBB1, ERBB2, ERBB3, ERBB 4, MYC, CCNDI, ESR I, PI3K, TOP 2A, APP, 3P, MGB1 and HPRT1 indicated good reaction performance and the calculated efficiencies of the reactions were within the range of $90.4-109.9 \%$ (Table III).

The isolated genomic DNA was confirmed to have high integrity and was seen as a single band on the electrophoretic gel. To confirm the high quality of the DNA, we performed a long PCR reaction on randomly chosen isolated material; the amplified $C Y P 2 D 6$ gene $(6,4 \mathrm{~kb})$ was seen as a single clear band. Each of the analysed targets was examined through a melt curve analysis and electrophoresis for the presence of primer dimers or unspecific bands; none were observed. No inhibition of the real-time PCR reaction was observed as the recorded $\mathrm{Cq}$ values for the $A P P$ and $3 P$ reference genes in the calibrator DNA, the DNA from healthy breast tissue, and the DNA supplied by Roche, the latter being free of qPCR inhibitors, had differences in Cqs $<0.5$.

The repeatability (intra-assay variation) and reproducibility (inter-assay variation) of the assay were measured as the average mRNA expression/gene dosage in pooled controlled mRNA/DNA on one plate (three reactions) or on four randomly selected separate plates, respectively. The assay was characterised by low intra-assay and inter-assay variation (data not shown).

The utility of the assay was verified with the use of the cell lines SK-BR-3 and MDA-MB-361, which had confirmed amplification of the genes $E R B B 2, M Y C, T O P 2 A$ and $E R B B 2$, respectively (27). Additionally, one copy of the $T O P 2 A$ gene is known to be deleted in MDA-MB-361 (31). All recorded values for ERBB2, MYC and TOP2A in the cell line SK-BR-3 were higher than the assumed cut-offs. The ERBB2 gene dosage in MDA-MB-361 was also higher than the cut-off and,
Table III. Reaction efficiencies.

\begin{tabular}{lrll}
\hline Gene & Efficiency & Slope & $\mathrm{R}^{2}$ \\
\hline$A P P$ & 109.6 & -3.112 & 0.997 \\
$3 P$ & 109.7 & -3.106 & 0.999 \\
$M Y C$ & 91.4 & -3.546 & 0.993 \\
$C C N D 1$ & 97.3 & -3.389 & 0.992 \\
ERBB1 & 96.6 & -3.406 & 0.994 \\
ERBB2 & 90.4 & -3.575 & 0.987 \\
ERBB3 & 91.7 & -3.536 & 0.987 \\
ERBB4 & 96.7 & -3.404 & 0.98 \\
ESR1 & 109.9 & -3.104 & 0.992 \\
PI3K & 90.5 & -3.572 & 0.98 \\
TOP2A & 96.8 & -3.4 & 0.992 \\
HPRT1 & 96.8 & -3.4 & 0.999 \\
$M G B 1$ & 108.2 & -3.14 & 0.964 \\
\hline
\end{tabular}

although the assay is not designed to measure deletions, the relative quantity of TOP $2 A$ was indeed decreased in this cell line (data not shown).

mRNA expression and gene dosage in clinical samples, cut-point determination and unsupervised data analysis. The relative quantities of gene expression and gene dosage were scaled to average. $M G B 1$ overexpression was recognised when there was at least 2-fold change of $M G B 1$ expression in comparison to the calibrator sample. The experimentally determined cut-off values were used in the evaluation of gene aberrations; as cut-offs, we used the most extreme values within the control samples.

Unsupervised hierarchical clustering was implemented to distinguish the gene aberration/expression profiles within the group of 157 EC patients. Out of the studied genes, ERBB4, $C C N D 1$ and ESR1 were excluded from the analysis as factors carrying little information. Instead, we included the RUNXI and $R A D 21$ mRNA expression levels, which were previously reported (32). Unsupervised analysis of gene aberrations and mRNA expression distinguished two major groups of tumours (clusters 1 and 2; Fig. 1). Patients within cluster 2 generally had increased gene dosages of $E R B B 3$ and elevated expression of $M G B 1, R U N X 1$ and RAD21.

Correlation of gene dosages and mRNA expression with clinical and pathological data. The gene dosages of the individual genes often correlated with each other. Particularly strong correlations occurred in the case of ERBBI and CCNDI $(\mathrm{p}=0.000002), E R B B 1$ and $E R B B 3(\mathrm{p}=0.000003), P I 3 K$ and $M G B 1(\mathrm{p}=0.00003), E R B B 3$ and TOP $2 A(\mathrm{p}=0.00005)$ and $E R B B 3$ and $P I 3 K(\mathrm{p}=0.00005)$. A higher stage of the disease was correlated with increased levels of $E R B B 2, E R B B 3$, ERBB 4, PI3K, MYC and TOP $2 A(\mathrm{p}=0.05, \mathrm{p}=0.003, \mathrm{p}=0.02$, $\mathrm{p}=0.02, \mathrm{p}=0.007, \mathrm{p}=0.04$, respectively). Histology type II was associated with elevated levels of ERBBI ( $\mathrm{p}=0.002), E R B B 3$ $(\mathrm{p}=0.01), P I 3 K(\mathrm{p}=0.05)$ and $M Y C(\mathrm{p}=0.02)$, and decreased expression of $M G B 1(\mathrm{p}=0.002)$. The presence of metastases was associated with higher $P I 3 K, M Y C$ and ESRl gene dosage 


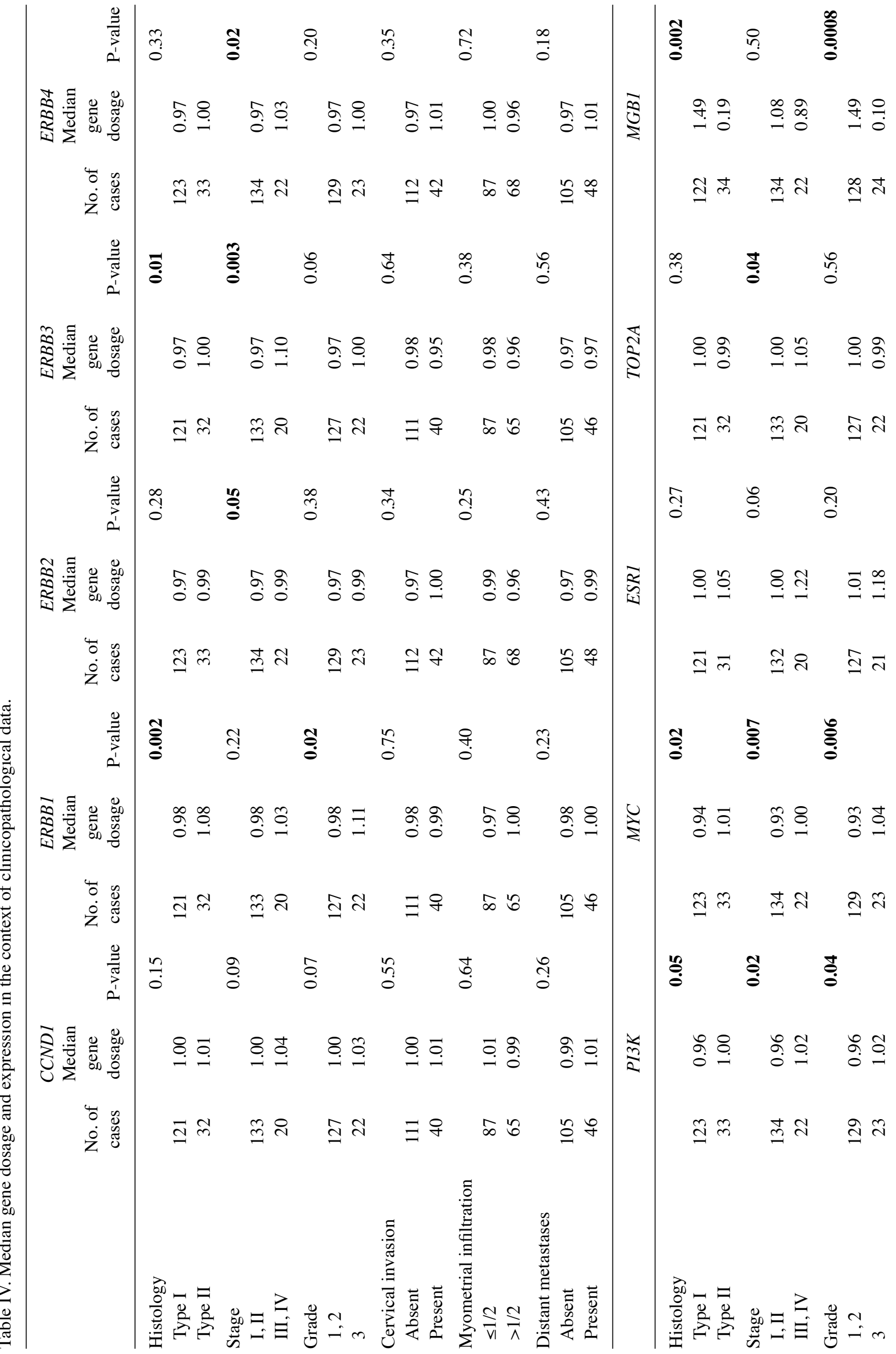




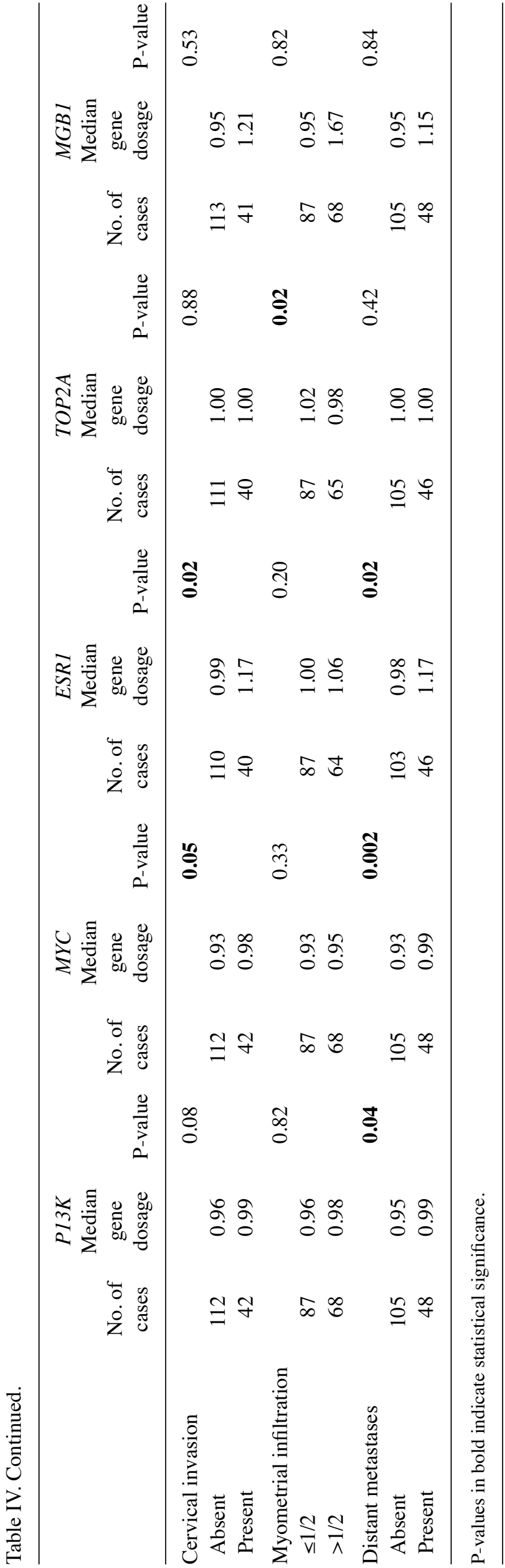

( $\mathrm{p}=0.04, \mathrm{p}=0.002, \mathrm{p}=0.02$, respectively), while a high grade was associated with increased levels of ERBBI $(\mathrm{p}=0.02)$, $P I 3 K(\mathrm{p}=0.04)$ and $M Y C(\mathrm{p}=0.006)$ and decreased level of $M G B 1(\mathrm{p}=0.0008)$. Cervical invasion was correlated with increased gene dosage of MYC $(\mathrm{p}=0.05)$ and ESRI $(\mathrm{p}=0.02)$. Myometrial infiltration was associated with elevated TOP $2 A$ levels ( $\mathrm{p}=0.02$; Table IV).

When we compared the groups of positive vs. negative samples within all the studied genes, particularly strong positive correlations were observed between the $E R B B 1$ status and histology type II $(\mathrm{p}=0.00001)$, the $E R B B I$ status and a higher grade $(\mathrm{p}=0.00005)$, the $E R B B 3$ status and a higher stage $(\mathrm{p}=0.00005)$ and the ESRl status and cervical invasion $(p=0.0003)$. Based on the analysis of separate genes, the gene dosage pattern of the ERBB family and its downstream effectors, PI3K and MYC, was analysed in more detail. Upregulation of the ERBB PI3K/Akt pathway was assumed whenever any of the $E R R B$ genes together with $P I 3 K$ and/or $M Y C$ were found to be amplified. The ERBB PI3K/Akt pathway was upregulated in $31(20 \%)$ of 156 cases. Activation of the pathway was positively correlated with a higher stage: $\mathrm{p}=0.001,21 / 134(15.7 \%)$ positive cases vs. $10 / 22(45.5 \%)$; grade: $p=0.001,19 / 129(14.7 \%)$ vs. $10 / 23$ (43.5\%); histological type II of the disease: $p=0.0003$, $17 / 123(13.8 \%)$ vs. $14 / 33(42.4 \%)$, as well as with the presence of metastases: $\mathrm{p}=0.02,16 / 105(15.2 \%)$ vs. $15 / 48$ (31.3\%).

Although the clusters were distinctive with generally higher gene dosages reported for cluster 2, there was no correlation between the results of the clustering and the clinicopathological characteristics.

Survival analysis. Of all the studied genes, with additional analysis of the ERBB PI3K/Akt pathway and hierarchical clustering, statistical significance with respect to the OS was reached only in the case of $E R B B 3$ and the generated clusters (both $\mathrm{p}=0.05$; Fig. $2 \mathrm{~A}$ and $\mathrm{B}$ ). The pattern of the remaining graphs, with $\mathrm{p}$-values $>0.05$, either had the expected tendency (shorter OS within the groups with gene amplification) or was inconclusive.

A univariate analysis was performed for all the clinicopathological data, the studied genes and the distinguished clusters. Stage, grade, PR status, ERBB3 status and the clusters correlated with shorter OS (all p $\leq 0.10$ ), and these parameters were included in a step-wise multivariate analysis, eventually yielding p-values of 0.01 and 0.13 for the stage, and $E R B B 3$, respectively. The hazard ratios for the stage and $E R B B 3$ were: 4.94 (95\% CI, 1.40-17.40), 3.27 (95\%CI, 0.68-15.65; Table V).

\section{Discussion}

Copy number variations belong to frequently found genetic alterations within cancerous tissues (33) and we have established a multimarker quantitative PCR (qPCR) platform to examine genes that may carry such aberrations and thus be of significance in endometrial cancer (EC) biology. The gene dosages of TOP $2 A, E R B B 1, E R B B 2, E R B B 3, E R B B 4$, $M Y C, C C N D 1, E S R 1, P I 3 K$ and $R A D 21$ were determined in fresh-frozen tumour samples using a SYBR-Green-based qPCR assay. Additionally, the RNA expression level of the $M G B 1$ gene was examined by RT-qPCR. 


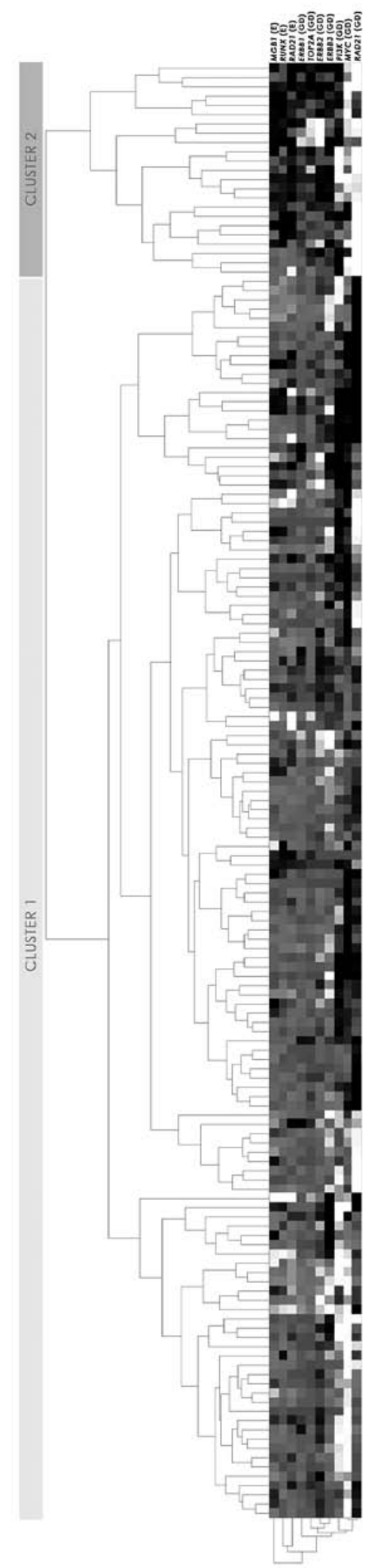

Figure 1. Results of unsupervised clustering of expression and gene dosage data from 157 endometrial carcinomas. Two major clusters were identified and recapitulated using the displayed set of 10 genes (E, expression data; GD, gene dosage data; black colour, high expression/gene dosage; grey, moderate; white, low).

The designed platform, exploited on a large sample size, proved to be a fast and simple way for direct analysis of the genes involved in uterine carcinogenesis. The results obtained
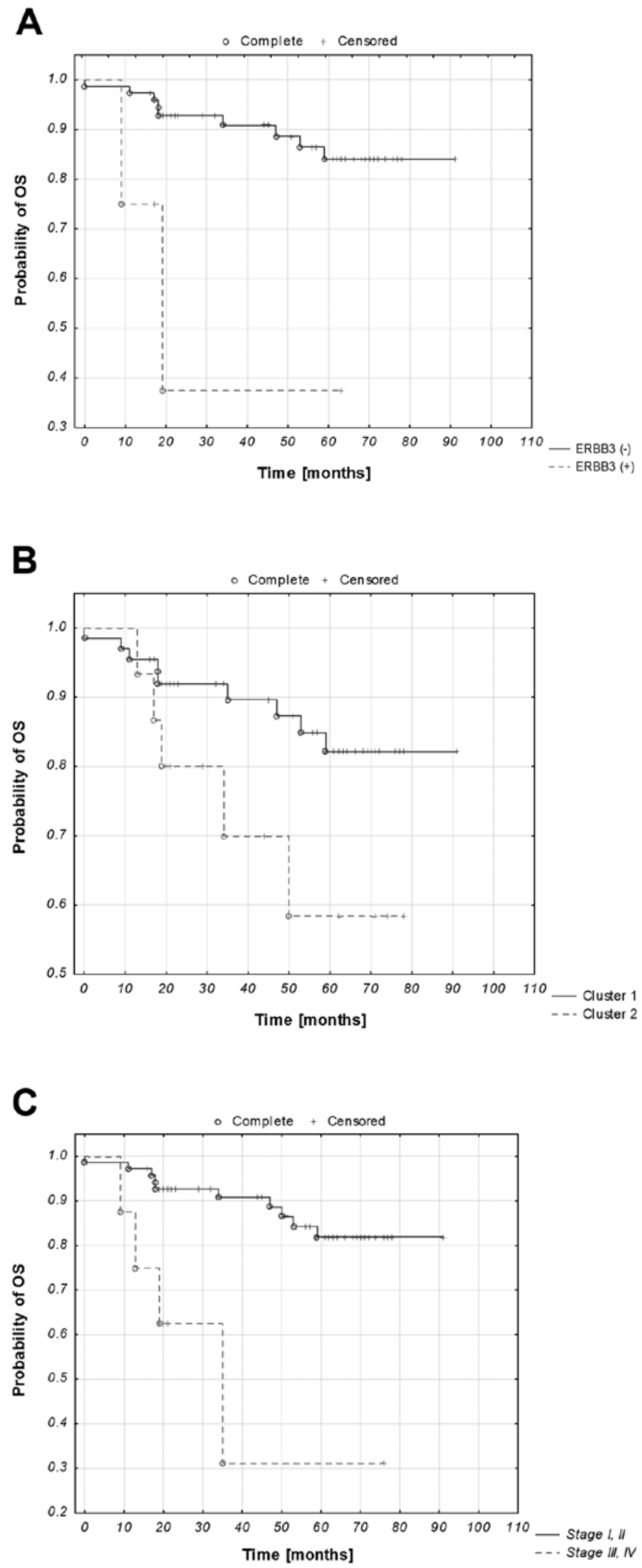

Figure 2. Kaplan-Meier curves presenting the overall survival of endometrial cancer patients stratified against (A) ERBB3 status ( $\mathrm{p}=0.05$ ), (B) two clusters $(\mathrm{p}=0.05),(\mathrm{C})$ the stage of the disease $(\mathrm{p}=0.01)$.

from versatile quality control tests showed good reaction performance, specificity of the designed primers, low intraassay and inter-assay variation, high DNA/RNA integrity and 
Table V. Univariate and multivariate analysis of clinicopathological and molecular parameters as prognostic factors in endometrial cancer patients.

\begin{tabular}{|c|c|c|c|c|c|c|}
\hline \multirow[b]{2}{*}{ Variable } & \multicolumn{3}{|c|}{ Univariate analysis } & \multicolumn{3}{|c|}{ Multivariate analysis } \\
\hline & HR & $95 \%$ CI & P-value & HR & $95 \% \mathrm{CI}$ & P-value \\
\hline Histology (type II vs. I) & 2.08 & $0.71-6.16$ & 0.18 & & & \\
\hline Stage (III, IV vs. I, II) & 6.02 & $1.84-19.66$ & 0.003 & 4.94 & $1.4-17.40$ & 0.01 \\
\hline Grade (3 vs. 1,2$)$ & 1.87 & $1.07-3.25$ & 0.03 & & NS & \\
\hline Obesity (present vs. absent) & 1.70 & $0.54-5.36$ & 0.36 & & & \\
\hline Cervical invasion (present vs. absent) & 1.45 & $0.46-4.59$ & 0.52 & & & \\
\hline Myometrial infiltration (present vs. absent) & 2.23 & $0.80-6.25$ & 0.13 & & & \\
\hline Metastases (present vs. absent) & 2.34 & $0.83-6.61$ & 0.11 & & & \\
\hline PgR status (positive vs. negative) & 0.33 & $0.10-1.05$ & 0.06 & & $N S$ & \\
\hline ER status (positive vs. negative) & 0.30 & $0.07-1.35$ & 0.12 & & & \\
\hline ERBB2 status (positive vs. negative) & 1.43 & $0.40-5.12$ & 0.58 & & & \\
\hline Clusters (2 vs. 1) & 2.57 & $0.87-7.54$ & 0.09 & & $N S$ & \\
\hline CCND1 status (positive vs. negative) & 1.53 & $0.23-10.19$ & 0.66 & & & \\
\hline ERBB1 status (positive vs. negative) & 1.05 & $0.27-4.13$ & 0.94 & & & \\
\hline ERBB2 status (positive vs. negative) & 2.72 & $0.78-9.55$ & 0.12 & & & \\
\hline ERBB3 status (positive vs. negative) & 5.31 & $1.24-22.90$ & 0.02 & 3.27 & $0.68-15.65$ & 0.13 \\
\hline ERBB4 status (positive vs. negative) & 1.08 & $0.25-4.61$ & 0.91 & & & \\
\hline PI3K status (positive vs. negative) & 0.82 & $0.11-5.90$ & 0.84 & & & \\
\hline MYC status (positive vs. negative) & 1.73 & $0.56-5.37$ & 0.34 & & & \\
\hline ESRl status (positive vs. negative) & 0.12 & $0.00-495.96$ & 0.62 & & & \\
\hline TOP2A status (positive vs. negative) & 2.12 & $0.51-8.80$ & 0.30 & & & \\
\hline MGB1 status (positive vs. negative) & 0.46 & $0.13-1.64$ & 0.23 & & & \\
\hline
\end{tabular}

quality, and thus low sample loss. We performed a utility verification using cancer cell lines, and the results, revealed the same aberrations that have been reported in the literature. The developed protocol allows for highly coherent gene dosage and transcriptomic measurements.

Mammaglobin 1 was first detected as a protein that is specifically overexpressed in breast cancer (34). Studies by Classen-Linke et al (35) demonstrated that this expression is not restricted to breast tissue; it may also be detected in the endometrium where it changes in a hormone-dependent manner. To the best of our knowledge, this is the first study to measure the MGBI RNA level in EC to understand its clinical significance. Our findings show that in EC, the loss of $M G B 1$ expression, not overexpression, strongly correlates with a higher tumour grade and is characteristic of histological type II.

Our results demonstrate that elevated $E R B B 1, E R B B 3$ and $M Y C$ gene dosages are correlated with more aggressive tumour behavior. Yeramian et al also found $M Y C$ to be deregulated in EC (10). However, these findings only involve gene expression. Data on $M Y C, E R B B 1$ and $E R B B 3$ copy number variations in $\mathrm{EC}$ are scarce or non-existent. Raeder et al analysed the amplifications of the 8q24 region in EC samples but did not observe any link between $M Y C$ copy number alterations and disease progression (36). Esteller et al studied the role of ERBBI in $\mathrm{EC}$, but they did not observe the ERBBI gene amplification in any of the cases studied (37). This may be due to the limitations of the method of differential PCR, which is much less sensitive than qPCR. Liang et al demonstrated that the overexpression of wild-type ERBB3 significantly increased the survival of $\mathrm{Ba} / \mathrm{F} 3$ cells (38), which is consistent with the proposed role of $E R B B 3$ as an oncogene. Our studies are also in agreement with the study of Saghir et al where ERBB3 overexpression was indicated as a characteristic typical for EC (39). It is well established in breast cancer that the amplification of genes belonging to the ERBB family results in the overexpression of tyrosine kinase receptors and our findings suggest that the same mechanism occurs in EC.

The borderline statistical significance of ERBB2 gene dosage in the context of the stage of the disease may be explained by the fact that $E R B B 2$ amplification was found to represent a rather rare event in EC (40). Nevertheless, Rolitsky et al found ERBB2 amplification to be correlated with type II EC, which supports our findings (41).

We demonstrated that the activation of the PI3K pathway is crucial in EC. These results are in agreement with studies by Salvesen et al (21) where amplification of a region including PIK3CA was significantly associated with unfavourable recurrence-free survival. Our data also indicated that increased ERBB/PI3K pathway gene dosage correlated with histological type II, higher stage and higher grade as well as the presence of metastases. 
The results described in the present study may facilitate the development of targeted therapies, developed to inhibit cellular signalling that leads to cell growth and proliferation in EC. Several tyrosine kinase receptor inhibitors are available, including ERBB1 inhibitors (gefitinib, erlotinib, lapatinib, cetuximab) and the ERBB2 inhibitor, trastuzumab. A phase II study on the use of cetuximab in recurrent EC is currently being carried out (42). There have also been trials on the efficacy of trastuzumab in ERBB2-positive EC patients. Although these studies did not report any activity $(43,44)$, further clinical evaluation of the use of trastuzumab or combination regiments may still be worth verifying as it is possible that this drug may be effective in a properly selected group of EC patients (45).

Although the clusters generated in the present study did not possess strikingly different clinical and histopathologic characteristics, cluster 2 in general did contain higher gene dosages with the exception of MYC and RAD21. Additionally, the survival analysis revealed a significantly shorter OS in cluster 2 , suggesting that this clustering distinction may in fact serve as a prognostic factor.

The key limitation of this study was the short follow-up period and lack of survival data for a considerable number of patients. As a result, the survival analyses rarely reached statistical significance, not only in the case of the studied genes but also in the analysis of the clinicopathological data. Nevertheless, even without statistical significance, almost all the studied potential markers retained the expected tendency (shorter OS for increased gene dosage/decreased expression), with few Kaplan-Meier curves being inconclusive due to a small sample size. The survival studies by Konecny et al (40), which were performed on a larger cohort of EC patients, did reach statistical significance, but these analyses focused mainly on type II EC, with a median follow-up of 83 months. The survival data for the cohort examined in the present study are still being collected. The prognostic value of the studied biomarkers is to be updated.

Another limitation of the present study is associated with tumour heterogeneity. The samples used for DNA/RNA isolation may not reflect the heterogeneity across a tumour, as they were excised manually from a single tumour region and only small tissue fragments were used. Perhaps a microdissection technique of formalin-fixed paraffin-embedded EC samples may be applied in future studies.

Our results demonstrate the utility of cancer genome characterisation in clinical specimens. The gene dosage pattern of the ERBB signalling network and the PI3K/Akt pathway differs in EC depending on the stage of the disease and the presence of metastases. The PI3K pathway is of particular importance in patients with aggressive disease. However, the prognostic value of the genes studied here remains to be determined in further survival studies. The results facilitate the understanding of the pathogenesis of EC and may also contribute to the development of more effective diagnostic tools. The potential for the application of this direct and rapid assay for EC patients is demonstrated in the case of the ERBB PI3K/Akt signalling pathway, whose status may provide additional diagnostic information. The developed highly sensitive and reproducible platform is feasible for EC characterisation.

\section{Acknowledgements}

The present study was supported by a grant from the National Science Centre (5715/B/P01/2010/38) and a grant from the Foundation for Polish Science Parent-Bridge Programme co-financed by the European Union within the European Regional Development Fund (DPS-424-5053/11). The present study was co-financed by the European Commission in the frame of the European Social Fund, by the European Social Fund, the State Budget, and the Pomorskie Voivodeship Budget according to the Operational Programme Human Capital, Priority VIII, Action 8.2, Under-action 8.2.2: 'Regional Innovative Strategy' within the system project of the Pomorskie Voivodeship 'InnoDoktorant-Scholarships for PhD students, Vth edition'. The publication was also financed by the European Social Fund as a part of the project 'Educators for the elite-integrated training program for $\mathrm{PhD}$ students, post-docs and professors as academic teachers at University of Gdansk' within the framework of the Human Capital Operational Programme, Action 4.1.1, improving the quality of educational offer of tertiary education institutions. The sponsors had no involvement in the present study design, collection, analysis and interpretation of the data, writing of the manuscript, or the decision to submit the manuscript for publication. The authors acknowledge Aleksandra Markiewicz for her support during the development of the multimarker platform and Bartosz Supernat for data visualisation, dendrogram generation, complete linkage computations and artwork adjustments. The manuscript was edited by Elsevier Language Editing Services.

\section{References}

1. Mirantes C,Espinosa I,Ferrer I,Dolcet X,Prat J and Matias-Guiu X: Epithelial-to-mesenchymal transition and stem cells in endometrial cancer. Hum Pathol 44: 1973-1981, 2013.

2. Wojciechowska U and Didkowska J: Cancer in Poland in 2010. Nowotwory 63: 197-216, 2013.

3. Bray F, Dos Santos Silva I, Moller H and Weiderpass E: Endometrial cancer incidence trends in Europe: underlying determinants and prospects for prevention. Cancer Epidemiol Biomarkers Prev 14: 1132-1142, 2005.

4. Bokhman JV: Two pathogenetic types of endometrial carcinoma. Gynecol Oncol 15: 10-17, 1983.

5. Horn LC, Meinel A, Handzel R and Einenkel J: Histopathology of endometrial hyperplasia and endometrial carcinoma: an update. Ann Diagn Pathol 11: 297-311, 2007.

6. Amant F, Moerman P, Neven P, Timmerman D, Van Limbergen E and Vergote I: Endometrial cancer. Lancet 366: 491-505, 2005.

7. Randall TC and Kurman RJ: Progestin treatment of atypical hyperplasia and well-differentiated carcinoma of the endometrium in women under age 40. Obstet Gynecol 90: 434-440, 1997.

8. Sherman ME, Sturgeon S, Brinton LA, et al: Risk factors and hormone levels in patients with serous and endometrioid uterine carcinomas. Mod Pathol 10: 963-968, 1997.

9. Ayhan A, Gultekin M and Dursun P (eds): Textbook of Gynaecological Oncology. Günes Publishing, Ankara, 2010.

10. Yeramian A, Moreno-Bueno G, Dolcet X, et al: Endometrial carcinoma: molecular alterations involved in tumor development and progression. Oncogene 32: 403-413, 2012.

11. Busmanis I, Ho TH, Tan SB and Khoo KS: p53 and bcl-2 expression in invasive and pre-invasive uterine papillary serous carcinoma and atrophic endometrium. Ann Acad Med Singapore 34: 421-425, 2005.

12. Caduff RF, Johnston CM and Frank TS: Mutations of the Ki-ras oncogene in carcinoma of the endometrium. Am J Pathol 146: 182-188, 1995.

13. Mutter GL, Lin MC, Fitzgerald JT, et al: Altered PTEN expression as a diagnostic marker for the earliest endometrial precancers. J Natl Cancer Inst 92: 924-930, 2000. 
14. Mutter GL, Boynton KA, Faquin WC, Ruiz RE and Jovanovic AS: Allelotype mapping of unstable microsatellites establishes direct lineage continuity between endometrial precancers and cancer. Cancer Res 56: 4483-4486, 1996.

15. Strissel PL, Ellmann S, Loprich E, et al: Early aberrant insulin-like growth factor signaling in the progression to endometrial carcinoma is augmented by tamoxifen. Int J Cancer 123 2871-2879, 2008.

16. Rose PG: Endometrial carcinoma. N Engl J Med 335: 640-649, 1996.

17. Onuma K, Dabbs DJ and Bhargava R: Mammaglobin expression in the female genital tract: immunohistochemical analysis in benign and neoplastic endocervix and endometrium. Int J Gynecol Pathol 27: 418-425, 2008

18. Grushko TA, Filiaci VL, Mundt AJ, et al: An exploratory analysis of HER-2 amplification and overexpression in advanced endometrial carcinoma: a gynecologic oncology group study. Gynecol Oncol 108: 3-9, 2008.

19. Zaczek A, Wełnicka-Jaśkiewicz M, Bielawski KP, et al: Gene copy numbers of $H E R$ family in breast cancer. J Cancer Res Clin Oncol 134: 271-279, 2008.

20. Zaczek A, Brandt B and Bielawski KP: The diverse signaling network of EGFR, HER2, HER3 and HER4 tyrosine kinase receptors and the consequences for therapeutic approaches. Histol Histopathol 20: 1005-1015, 2005.

21. Salvesen HB, Carter SL, Mannelqvist M, et al: Integrated genomic profiling of endometrial carcinoma associates aggressive tumors with indicators of PI3 kinase activation. Proc Natl Acad Sci USA 106: 4834-4839, 2009.

22. Ponzielli R, Katz S, Barsyte-Lovejoy D and Penn LZ: Cancer therapeutics: targeting the dark side of Myc. Eur J Cancer 41: 2485-2501, 2005.

23. Semczuk A and Jakowicki JA: Alterations of pRb1-cyclin D1-cdk4/6-p16 $6^{\mathrm{INK} 4 \mathrm{~A}}$ pathway in endometrial carcinogenesis Cancer Lett 203: 1-12, 2004

24. Tsiambas E, Alexopoulou D, Lambropoulou S, Gerontopoulos K, Karakitsos P and Karameris A: Targeting topoisomerase IIa in endometrial adenocarcinoma: a combined chromogenic in situ hybridization and immunohistochemistry study based on tissue microarrays. Int J Gynecol Cancer 16: 1424-1431, 2006.

25. Pecorelli S: Revised FIGO staging for carcinoma of the vulva, cervix, and endometrium. Int J Gynaecol Obstet 105: 103-104, 2009.

26. Consultation WE: Appropriate body-mass index for Asian populations and its implications for policy and intervention strategies. Lancet 363: 157-163, 2004.

27. Jönsson G, Staaf J, Olsson E, et al: High-resolution genomic profiles of breast cancer cell lines assessed by tiling BAC array comparative genomic hybridization. Genes Chromosomes Cancer 46: 543-558, 2007.

28. Vandesompele J, De Preter K, Pattyn F, et al: Accurate normalization of real-time quantitative RT-PCR data by geometric averaging of multiple internal control genes. Genome Biol 3 : research0034, 2002

29. Hellemans J, Mortier G, De Paepe A, Speleman F and Vandesompele $\mathrm{J}$ : qBase relative quantification framework and software for management and automated analysis of real-time quantitative PCR data. Genome Biol 8: R19, 2007.

30. McShane LM, Altman DG, Sauerbrei W, et al: REporting recommendations for tumor MARKer prognostic studies (REMARK) Nat Clin Pract Oncol 2: 416-422, 2005.
31. Järvinen TA, Tanner M, Rantanen V, et al: Amplification and deletion of topoisomerase II $\alpha$ associate with ErbB-2 amplification and affect sensitivity to topoisomerase II inhibitor doxorubicin in breast cancer. Am J Pathol 156: 839-847, 2000.

32. Supernat A, Lapińska-Szumczyk S, Sawicki S, Wydra D, Biernat W and Zaczek AJ: Deregulation of RAD21 and RUNX1 expression in endometrial cancer. Oncol Lett 4: 727-732, 2012.

33. Inaki K and Liu ET: Structural mutations in cancer: mechanistic and functional insights. Trends Genet 28: 550-559, 2012.

34. Watson MA and Fleming TP: Mammaglobin, a mammary-specific member of the uteroglobin gene family, is overexpressed in human breast cancer. Cancer Res 56: 860-865, 1996.

35. Classen-Linke I, Moss S, Gröting K, Beier HM, Alfer J and Krusche CA: Mammaglobin 1: not only a breast-specific and tumour-specific marker, but also a hormone-responsive endometrial protein. Histopathology 61: 955-965, 2012.

36. Raeder MB, Birkeland E, Trovik J, et al: Integrated genomic analysis of the 8q24 amplification in endometrial cancers identifies $A T A D 2$ as essential to $M Y C$-dependent cancers. PLoS One 8: e54873, 2013.

37. Esteller M, García A, Martínez i Palones JM, Cabero A and Reventós J: Detection of c-erbB-2/neu and fibroblast growth factor-3/INT-2 but not epidermal growth factor receptor gene amplification in endometrial cancer by differential polymerase chain reaction. Cancer 75: 2139-2146, 1995.

38. Liang H, Cheung LW, Li J, et al: Whole-exome sequencing combined with functional genomics reveals novel candidate driver cancer genes in endometrial cancer. Genome Res 22: 2120-2129, 2012

39. Saghir FS, Rose IM, Dali AZ, Shamsuddin Z, Jamal AR and Mokhtar NM: Gene expression profiling and cancer-related pathways in type I endometrial carcinoma. Int J Gynecol Cancer 20: 724-731, 2010.

40. Konecny GE, Santos L, Winterhoff B, et al: HER2 gene amplification and EGFR expression in a large cohort of surgically staged patients with nonendometrioid (type II) endometrial cancer. Br J Cancer 100: 89-95, 2009.

41. Rolitsky CD, Theil KS, McGaughy VR, Copeland LJ and Niemann TH: HER-2/neu amplification and overexpression in endometrial carcinoma. Int J Gynecol Pathol 18: 138-143, 1999.

42. Tsikouras P, Bouchlariotou S, Vrachnis N, et al: Endometrial cancer: molecular and therapeutic aspects. Eur J Obstet Gynecol Reprod Biol 169: 1-9, 2013

43. Fleming GF, Sill MW, Darcy KM, et al: Phase II trial of trastuzumab in women with advanced or recurrent, HER2-positive endometrial carcinoma: a Gynecologic Oncology Group study. Gynecol Oncol 116: 15-20, 2010.

44. Santin AD, Bellone S, Roman JJ, McKenney JK and Pecorelli S: Trastuzumab treatment in patients with advanced or recurrent endometrial carcinoma overexpressing HER2/neu. Int J Gynaecol Obstet 102: 128-131, 2008.

45. Santin AD: Letter to the Editor referring to the manuscript entitled: 'Phase II trial of trastuzumab in women with advanced or recurrent HER-positive endometrial carcinoma: a Gynecologic Oncology Group study' recently reported by Fleming et al, (Gynecol Oncol., 116;15-20;2010). Gynecol Oncol 118: 95-96; author reply 96-97, 2010. 